\title{
Comunicação
}

[Communication]

\section{Estudo da correlação da densitometria óssea e do perímetro diafisário medial do terceiro metacarpiano em equinos}

\author{
[Correlation study on equine bone densitometry and third metacarpus diaphyseal perimeter]
}

\author{
A.C.S. Aguiar ${ }^{1}$, V.M.V. Machado ${ }^{2}$, R.V. Santos ${ }^{3}$, J.N.P. Puoli Filho ${ }^{2}$, M.D.S. Mota ${ }^{2}$, L.C. Vulcano ${ }^{2}$ \\ ${ }^{1}$ Aluna de graduação - FMVZ - UNESP - Botucatu, SP \\ ${ }^{2}$ Faculdade de Medicina Veterinária e Zootecnia - UNESP - Botucatu, SP \\ ${ }^{3}$ Aluna de pós-graduação - Faculdade de Medicina Veterinária e Zootecnia - UNESP - Botucatu, SP
}

Os ossos são compostos por matriz orgânica de colágeno e por cristais inorgânicos, como cálcio e fosfato (Okuno et al., 2003; Osbjorn e Lieberman, 2004). A deposição de minerais nos ossos está relacionada com sua capacidade biomecânica e sofre influência de fatores como idade, nutrição e treinamento (Reichmann et al., 2004). Já a resistência dos ossos varia de acordo com a densidade mineral, as características trabeculares, a porosidade, a duração da força aplicada e as propriedades biomecânicas (Bogna et al., 2005).

O osso é um tecido dinâmico que sofre ajustes contínuos de sua massa e arquitetura, decorrentes do estímulo mecânico promovido pelas atividades físicas (Kemper et al., 2002). Contudo, durante o treinamento de animais atletas, a sobrecarga óssea pode levar ao aparecimento de falhas microscópicas e ao acúmulo de microfraturas. Nestes casos, há um aumento temporário da porosidade, visto que há mobilização de reservas para reparação óssea, o que pode conduzir ao enfraquecimento ósseo e aumentar o risco de fratura (Danova et al., 2003).

O terceiro osso metacarpiano é frequentemente utilizado em pesquisas de densitometria óssea em equinos, devido à posição anatômica acessível, ao formato ósseo, à distribuição uniforme de tecidos moles e à alta susceptibilidade às injúrias locomotoras, haja vista a magnitude das forças compressivas às quais é submetido (Adams, 1996; Okuno et al., 2003).

Recebido em 1 de abril de 2011

Aceito em 3 de abril de 2012

*Autor para correspondência (corresponding author)

E-mail: vaniamvm@fmvz.unesp.br
A densidade óssea é considerada um parâmetro biofísico de grande valor experimental e clínico na avaliação do processo de mineralização óssea (Louzada, 1990). Assim, várias técnicas não invasivas são estudadas visando avaliar o tecido ósseo em animais jovens, em período de crescimento, e em animais adultos com protocolos de treinamento de baixa e alta intensidade (Lepage et al., 1998; Riggs, 1999; McCarthy e Jeffcott, 1992).

Alguns criadores de animais para atividades esportivas acreditam que animais com maior espessura da região do metacarpo possuem menor risco de fratura. Considerando a alta frequência de lesões ósseas, principalmente as fissuras do terceiro osso metacarpiano de animais destinados às atividades esportivas, este estudo teve como objetivo correlacionar as técnicas de densitometria óssea, ultrassonografia quantitativa (QUS) e tomografia computadorizada, com o perímetro do terceiro osso metacarpiano de equinos post-mortem.

Foram utilizados quatorze membros torácicos de equinos post-mortem, os quais não apresentavam sinais radiográficos e tomográficos compatíveis com alterações ósseas do terceiro metacarpiano. Os animais foram provenientes do Departamento de Patologia Animal, da Faculdade de Medicina Veterinária e Zootecnia da UNESP, campus de Botucatu. Para a padronização do estudo, as mensurações dos perímetros foram realizadas na porção medial da diáfise do terceiro metacarpiano, e a densitometria óssea foi feita no mesmo local, porém apenas na região dorsal. 
A mensuração da densidade óssea foi obtida por meio do aparelho de ultrassonografia quantitativa (Sunlight EQUS, Sunlight Medical Ltd, Israel, 2002), com auxílio de um transdutor microlinear CM-probe, com frequência de 1,2 MHz, e do tomógrafo helicoidal (Shimathzu, modelo SCT7800 TC), ambos disponíveis no setor de diagnóstico por imagem da Faculdade de Medicina Veterinária e Zootecnia da UNESP, campus Botucatu.

Após a calibração do aparelho de ultrassonografia quantitativa, aplicou-se óleo mineral na superfície das peças para beneficiar a condução das ondas ultrassonográficas. A probe foi posicionada longitudinalmente à superfície dorsal da região medial da diáfise do terceiro metacarpiano. A mensuração da velocidade das ondas foi realizada pelo sistema computacional com tecnologia Omnisense (Sunlight Medical Ltd, Israel, 2002).

A probe do aparelho emite ondas acústicas pulsáteis e permite a avaliação óssea sob partes moles de até $9 \mathrm{~mm}$ de espessura. O software utilizado calcula a velocidade ultrassonográfica (SOS) a partir da menor velocidade de propagação das ondas através dos ossos. Cada ciclo para avaliação da QUS leva cerca de 20 segundos e o computador registra de três a cinco ciclos seguidos, para obter resultados significativos.

As imagens tomográficas foram adquiridas posicionando-se os membros na mesa tomográfica, em decúbito dorsal, sendo realizados cortes axiais de $2 \mathrm{~mm}$ da região em estudo. Foram utilizados, em todas as peças, os valores de $120 \mathrm{Kv}, 130 \mathrm{MA}$ e a velocidade de rotação do tubo de um segundo em cortes retos. Por meio do software Voxar 3D (Voxar 3D, versão 6.3, Barco, 2009), foram demarcadas áreas circulares de $0,4 \mathrm{~cm}^{2}$ que incluíam a cortical óssea e se estendiam até, no máximo, $0,5 \mathrm{~cm}$ em direção à região medular. A densitometria óssea foi realizada nos cinco cortes tomográficos sequenciais e obtida pela média aritmética destes.

A análise estatística utilizada no estudo foi a correlação de Pearson realizada por meio do software Sigma Stat 3.5, além de estatística descritiva.

A média e os valores mínimo e máximo do perímetro e da densitometria óssea da região proposta, por meio das duas técnicas, estão apresentados na Tab. 1.

Tabela 1. Resultados referentes à média e aos valores mínimo e máximo do perímetro, velocidade da onda (SOS) obtida pela ultrassonografia quantitativa e densidade óssea na tomografia computadorizada da porção dorsal da região diafisária medial do terceiro metacarpiano

\begin{tabular}{lccc}
\hline \multicolumn{1}{c}{ Métodos } & Média & Valor mínimo & Valor máximo \\
\hline Perímetro (cm) & $16,49 \mathrm{~cm}$ & $15,5 \mathrm{~cm}$ & $17,5 \mathrm{~cm}$ \\
Velocidade da onda (SOS) & 4014,21 & 4148 & 3777 \\
$\begin{array}{l}\text { Densidade na tomografia } \\
\text { computadorizada }\end{array}$ & $1861,94 \mathrm{HU}$ & $1558,32 \mathrm{HU}$ & $2014,88 \mathrm{HU}$ \\
\hline
\end{tabular}

A Fig. 1 demonstra um dos cortes axiais obtidos pela tomografia computadorizada e demarca o local onde foi realizada a densitometria óssea.

A correlação de Pearson não apresentou resultados estatisticamente significativos entre o perímetro da região diafisária medial do terceiro metacarpiano e a densitometria óssea realizada pelo aparelho de ultrassonografia quantitativa, como demonstra a Fig. $2(\mathrm{P}=0,16)$.

A correlação entre a mensuração da densidade óssea realizada no tomógrafo e o perímetro da região medial da diáfise do terceiro metacarpiano também não foi significativa, segundo a correlação de Pearson, como demonstra a Fig. 3 $(\mathrm{P}=0,86)$. 


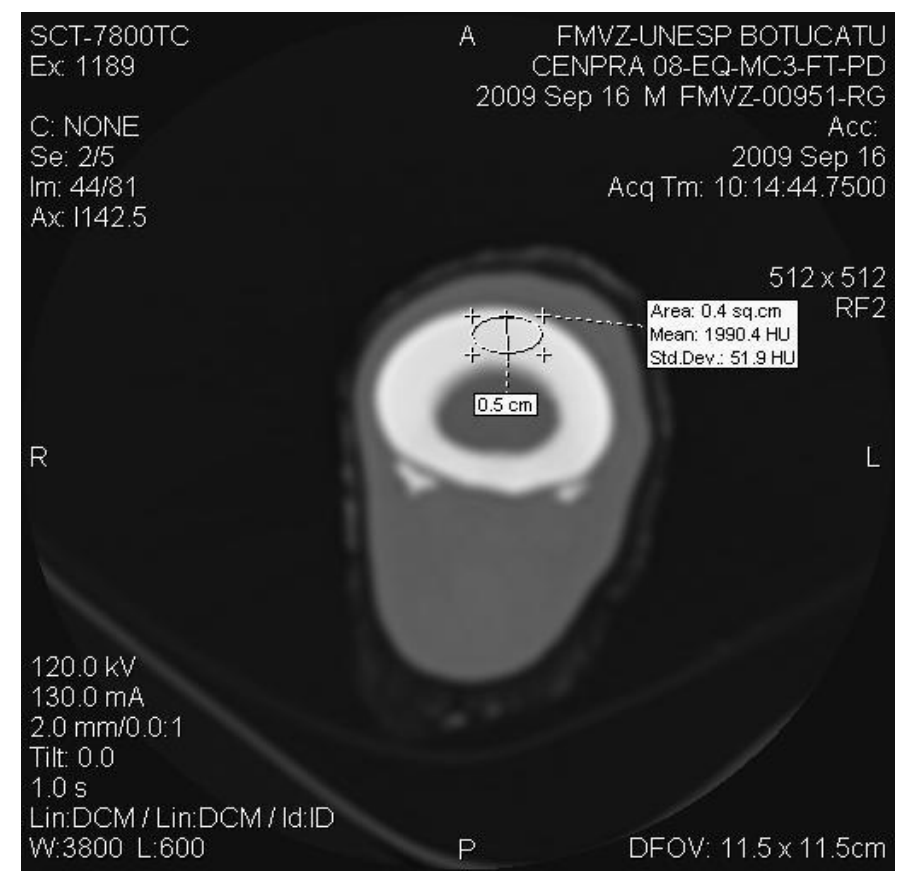

Figura 1. Corte tomográfico axial da região medial do terceiro metacarpiano demonstrando a espessura de $0,5 \mathrm{~cm}$ da cortical. A elipse indica a área onde foi realizada a densitometria com valor de 1990,4 HU e desvio-padrão de 51,9 HU.

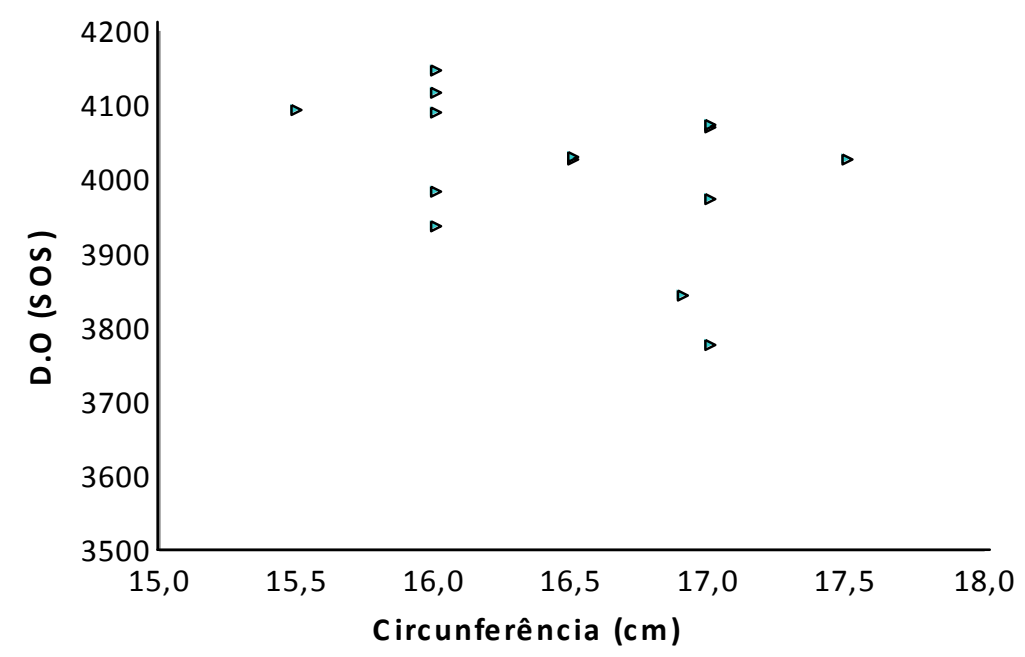

Figura 2. Dispersão com os valores dos 14 membros de equinos post-mortem referentes ao perímetro (cm) versus a densitometria óssea (DO) por meio da mensuração da velocidade da onda (SOS) obtida pela ultrassonografia quantitativa. 


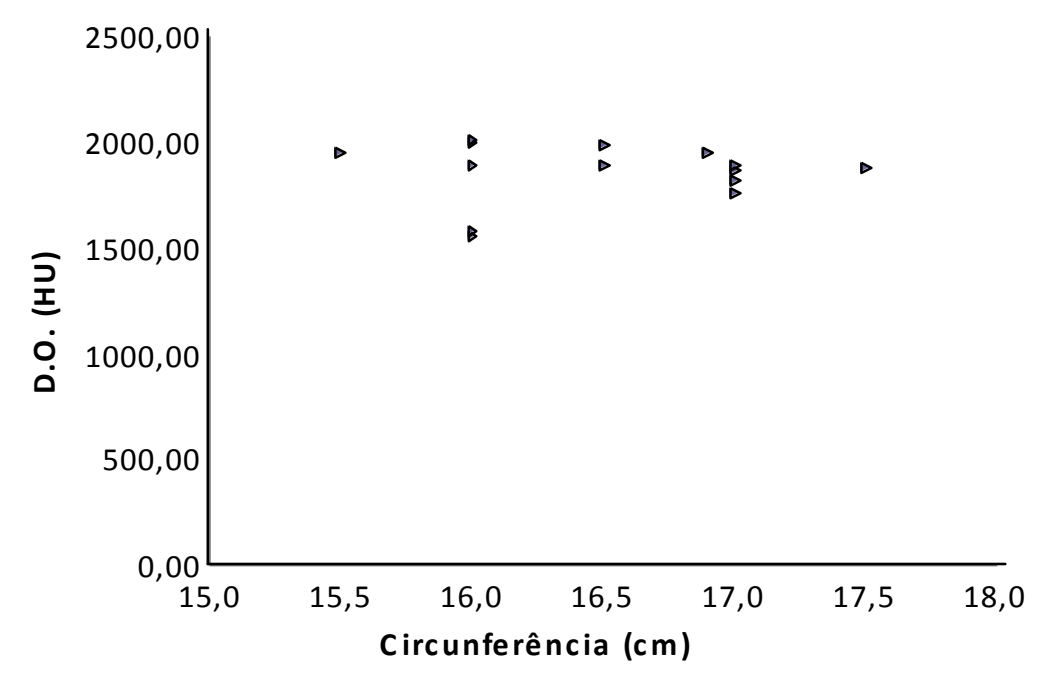

Figura 3. Dispersão com valores dos 14 membros de equinos post-mortem referentes ao perímetro (cm) versus a densitometria óssea obtida pela tomografia computadorizada (HU).

Os resultados não demonstraram correlação entre o perímetro do terceiro metacarpiano e a densitometria óssea, tanto pela QUS quanto pela tomografia. O fato de não haver conhecimento prévio de variáveis que influenciam na mineralização óssea, como idade, alimentação e treinamento recebido pelos animais, dificultou a possibilidade de inferir propostas para abordar a variância das variáveis em questão (Reichmann et al., 2004).

\begin{abstract}
Não há relatos na literatura de trabalhos que utilizem este tipo de correlação, para comparar as variáveis em questão. Sugere-se a realização de experimentos padronizados para averiguar a confiabilidade do uso do perímetro da região diafisária medial do terceiro metacarpiano como método de seleção de animais com menor risco de fratura.
\end{abstract}

Palavras-chave: ultrassonografia quantitativa, equinos, terceiro metacarpiano

\begin{abstract}
In this study, 14 "post mortem" equine metacarpal bones were evaluated to correlate the medial III metacarpus perimeter to dorsal bone density of the same region using Computed Tomography and Quantitative Ultrasound (QUS). According to Pearson's test, there were not correlations between these variables. Considering there are few bone densitometry studies in horses and there are many variables that can interfere with bone mineralization, new studies on equine bone density applying standard variables are recommended.
\end{abstract}

Keywords: quantitative ultrasound, equine, third metacarpal

\section{REFERÊNCIAS}

ADAMS, O.R. The clinical equine. In: Lameness in horses. Philadelphia: Lea \& Febiger, 1996. chap. 9, p.285-326.

BOGNA, D.; PLUSKIEWICZ, W. Quantitative ultrasound in diagnosis of metabolic bone disease. Curr. Med. Imaging Rev., v.1, p.67-74, 2005.
DANOVA, N.A.; COLOPY, S.A.; RADTKE, C.L. et al. Degradation of bone structural by accumulation and coalescence of microcracks. Bone, v.33, p.197-205, 2003.

KEMPER, H.C.G.; BAKKER, I.; TWISK, J.W.R. et al. Validation of a physical activity questionnaire to measure the effect of mechanical strain on bone mass. Bone, v.30, p.799-804, 2002. 
LEPAGE, O.M.; CARSTANJEN, B.; UEBELHART, D. Non-invasive assessment of equine bone: an update. Vet. J., v.161, p.10-23, 2001.

LEPAGE, O.M.; WHITTON, C.; DANIELLI, A. Multisite quantitative ultrasound measurements in horse: preliminary results for metacarpal bone assessment. Bone, v.13, p.214-218, 1998.

LOUZADA, M.J.Q.; XAVIER, C.A.M.; MARQUES NETO, J.F. Ação da calcitonina na resolução de perfurações ósseas em coelhos: controle fotodensimétrico e histológico. Rev. Bras. Endocrinol., v.30, p.111-116, 1990.

McCARTHY, R.N.; JEFFCOTT, L.B. Effects of treadmill exercise on cortical bone in the third metacarpus of young horses. Res. Vet. Sci., v.52, p.28-37, 1992.
OKUNO, E.; FRATIN, L. Desvendando a física do corpo humano: biomecânica. In: São Paulo:Manole, 2003. p.142-150.

OSBJORN, M.P.; LIEBERMAN, D.E. The aging of Wolff's law: ontogeny and responses to mechanical loading in cortical bone. Yearbook Phys. Anthropol., v.47, p.63-99, 2004.

REICHMANN, P.; MOURE, A.; GAMBA, H.R. Bone mineral content of the third metacarpal bone in quarter horse foals from birth to one year of age. J. Equine Vet. Med. v.24, p.391-396, 2004.

RIGGS, C.M. The etiopathogenesis of parasagital fractures of the distal condyles of the third metacarpal and third metatarsal bone. Equine Vet. J., v.31, p.116-120, 1999. 